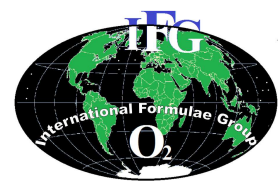

\title{
Typologie spatiale de la végétation sahélienne en relation avec les indicateurs de dégradation au Burkina Faso
}

\author{
Abel KADEBA ${ }^{1 *}$, Oumarou SAMBARE ${ }^{1,2}$, Soungalo SOULAMA ${ }^{1,3}$, \\ Adjima THIOMBIANO ${ }^{1}$, Marco SCHMIDT ${ }^{4,5,6}$ et Joseph Issaka BOUSSIM ${ }^{1}$ \\ ${ }^{1}$ Laboratoire de Biologie et Ecologie Végétales, Université de Ouagadougou, \\ 03 BP 7021 Ouagadougou 03, Burkina Faso. \\ ${ }^{2}$ Institut des Sciences, 01 BP 1757 Ouagadougou 01, Burkina Faso. \\ ${ }^{3}$ Centre National de la Recherche Scientifique et Technologique, Institut de l'Environnement et de Recherches \\ Agricoles, Département Productions Forestières, 03 BP 7047 Ouagadougou 03, Burkina Faso. \\ ${ }^{4}$ Senckenberg Research Institute, Dept. Botany and mol. Evolution, Senckenberganlage 25, \\ 60325 Frankfurt am Main, Germany. \\ ${ }^{5}$ J. W. Goethe University, Institute of Ecology, Evolution and Diversity. \\ Max-Von-Laue-Str. 9, 60438 Frankfurt am Main. Germany. \\ ${ }^{6}$ Biodiversity and Climate research Center (BiK-F). Senckenberganlage 25, \\ 60325 Frankfurt am Main, Germany. \\ *Auteur correspondant; E-mail: kadebab@yahoo.fr Tel: +22676010549
}

\section{RESUME}

La variation spatiale du couvert végétal constitue un des déterminismes majeurs de la qualité des écosystèmes. Elle influence la physionomie spatiale de la végétation ainsi que les mutations des ressources naturelles. L'objectif de ce travail est d'examiner la typologie spatiale de la végétation sahélienne selon le taux de couverture végétale en décrivant les glacis selon le stade de dégradation à travers une caractérisation fonctionnelle et structurale. La variation spatiale de la couverture végétale a été déterminée par utilisation d'une image satellitaire de type Modis appuyée d'observations visuelles et d'investigations de terrain. Erdas Imagine 9.2 et ArcGIS.10 ont été utilisés pour la cartographie synchronique de l'indice Normalisé Différentiel de Végétation (NDVI). Le taux de couverture végétale a été visuellement apprécié sur le terrain. Des relevés phytosociologiques et dendrométriques ont été faits et des variables environnementales ont été examinées et caractérisées. Au terme de cette étude, trois stades de dégradation basés sur le taux de couverture végétale ont été définis: le stade très dégradé (NDVI faible et couverture végétale $<10 \%)$, le stade moyennement dégradé (NDVI moyen et $10 \%$ < couverture végétale < 30\%) et le stade peu dégradé (NDVI élevé et couverture végétale $\geq 30 \%$ ). Les analyses multivariées montrent une importante dissimilarité entre les stades de dégradation des glacis. La caractérisation comparative des variables a révélé des différences significatives. L'analyse statistique en composantes principales (ACP) basée sur une approche corrélative a permis la mise en évidence des meilleures variables indicatrices de chaque stade de dégradation. Cette étude constitue un outil d'aide à la caractérisation des stades de dégradation des glacis sahéliens, étape importante pour le suivi de la désertification pour un aménagement durable des écosystèmes.

(C) 2014 International Formulae Group. All rights reserved.

Mots clés: Images satellitaires, couverture végétale, stades de dégradation, variables indicatrices, aménagement durable, Burkina Faso. 


\section{INTRODUCTION}

La zone sahélienne de l'Afrique occidentale est reconnue pour son problème de sécheresse et de désertification (Agnew et al., 2000) qui constituent la problématique environnementale la plus préoccupante du $\mathrm{XXI}^{\text {ème }}$ siècle (Benslimane et al., 2008). Les investigations sur la désertification ont montré ses manifestations et son impact chronique sur les ressources naturelles à travers la détérioration du milieu (Jacqueminet, 1991). Ce qui a motivé les multiples études sur ce phénomène dans le monde scientifique dans le souci d'inverser la tendance et de proposer des solutions durables (Jauffret, 2001; Miguel et al., 2011; Morsli et al., 2004; Rakotoarimanana et al., 2008; Maitima et al., 2004; Grouzis, 1983). Ces études ont mentionné que l'utilisation des terres et le climat sont les principales causes de l'intensification de la désertification. Selon la Conférence des Nations Unies sur l'Environnement et le Développement, la désertification se définit comme la dégradation des terres dans les zones arides, semi-arides et subarides sous l'effet de divers facteurs incluant les variations climatiques et les activités humaines (Cornet, 2002). La dégradation des terres, caractérisée par sa complexité, a été largement discutée (Brabant et al., 2010; Al-Awadhi et al., 2005). De nombreuses définitions ont été proposées et toutes s'accordent sur le fait que la dégradation des terres est l'incapacité actuelle et future des terres à produire (Fatunbi et al., 2008). La dégradation des terres a des effets néfastes car elle constitue un obstacle à la sécurité alimentaire, aux programmes d'aides internationaux, à l'économie nationale pour le développement et aux stratégies de conservation des ressources naturelles (Wessels et al., 2004). Elle accélère conséquemment la pauvreté dans les pays en développement en augmentant la proportion des populations humaines vulnérables (Peter et al., 2008).

La partie nord du Burkina Faso est sous un climat sahélien. La pluviométrie est faible avec des températures variables. Un élevage et une agriculture extensifs et intensifs y sont pratiqués. Ces différents facteurs imposent aux glacis sahéliens une dégradation de plus en plus prononcée de leurs ressources naturelles accentuant l'érosion hydrique et éolienne (Bénié et al., 2005).

De multiples travaux, par le biais de différents programmes et projets ont été menés dans le Sahel Burkinabé (Kagambèga et al., 2011; Kiéma et al., 2008). Les résultats liés à ces différents travaux restent en général insatisfaisants du fait du manque de suivi d'une part et de la connaissance incomplète des conditions écologiques particulières du milieu d'autre part. Selon Brabant (1996), il existe une succession de stades de dégradation du couvert végétal allant du stade sévèrement dégradé au stade non dégradé et du stade irréversible au stade réversible. Ainsi, suivant le stade de dégradation, l'intervention peut être une reconstitution de la banque de semences ou le stock de matières organiques et de micro-organismes, un reconditionnement des sols ou une réactivation de leur fonctionnement hydrique (Aronson et al., 1995). Les travaux antérieurs ont omis ce diagnostic du stade de dégradation, d'où des insuffisances conformément aux résultats escomptés. Pour combler ces insuffisances, il apparait impérieux de diagnostiquer le stade de dégradation d'un écosystème avant d'y entreprendre toute activité de restauration. La connaissance du stade de dégradation nécessite la mise en place d'indicateurs visuels, pertinents, faciles à mesurer et permettant d'indiquer à toute période le stade de dégradation d'un écosystème donné afin d'y asseoir l'intervention appropriée (Roselt / Oss, 2004).

L'objectif général de cette étude est de diagnostiquer et de caractériser différents stades de dégradation des glacis sahéliens à travers une méthode synchronique afin d'aider à conduire les efforts de restauration vers plus de succès. Cette étude vise spécifiquement (i) à proposer une typologie de la végétation sahélienne à travers l'imagerie satellitaire 
couplée aux données de terrain (ii) à appréhender les caractéristiques fonctionnelles et structurales des stades de dégradation des glacis et (iii) à proposer des indicateurs de dégradation observables propres à chaque stade de dégradation. L'hypothèse qui soustend cette étude stipule que la variation spatiale de la couverture végétale est une expression de différents stades de dégradation ayant chacune des caractéristiques structurales, fonctionnelles et des indicateurs de dégradation bien propres. En effet, le suivi du couvert végétal ainsi que les mutations des écosystèmes est une issue clé pour la lutte contre la dégradation et par conséquent la conservation des habitats (Kosmidou et al., 2014).

\section{MATERIEL ET METHODES \\ Zone d'étude}

Cette étude a été conduite dans le bassin versant du Gourouol dans la province du Séno situé au Sahel-strict (Guinko, 1984) du Burkina Faso (Figure 1). D'une superficie d'environ 390000 ha, le bassin versant du Gourouol, est confiné entre les latitudes $14^{\circ} 3^{\prime}-14^{\circ} 10^{\prime} \mathrm{N}$ et les longitudes $0^{\circ} 5^{\prime}-0^{\circ} 11^{\prime} \mathrm{E}$ (Kiéma et al., 2008). Le réseau hydrographique est formé par la rivière Gourouol et ses affluents que sont le Féléol et le Goudébo (Barral, 1967). La moyenne pluviométrique annuelle y varie entre 200 et $400 \mathrm{~mm}$. Le bassin versant du Gourouol se caractérise par deux saisons distinctes: une saison sèche de 9 mois (octobre-juin) aggravée par l'harmattan qui souffle en permanence de l'Est au Nord-Est et une saison pluvieuse de 3 mois (juillet-septembre) (Kiéma et al., 2008). La population pratique principalement l'agriculture et l'élevage (Bénié et al., 2005). Il y existe plusieurs unités géomorphologiques qu'on peut regrouper en trois: les glacis, les dunes de sables et les basfonds qui occupent respectivement $60 \%, 30 \%$ et $10 \%$ en superficies (Grouzis, 1984). La végétation est alternée de steppes dominées par les Fabaceae-Mimosoideae et de brousses tigrées plus ou moins érodées (Thiombiano et al., 2012).

\section{Collecte de données \\ Données satellitaires}

Une image MODIS (Moderate

resolution Imaging Spectroradiometer), acquise en octobre 2010 a été utilisée. Le choix de l'image MODIS s'explique par sa disponibilité d'une part et parce que ce type d'image est plus approprié à l'analyse de la variation du taux de couverture végétale d'autre part. Le mois d'octobre a été préféré parce qu'il correspond à la période active de la végétation. Pendant cette période, les superficies cultivées sont faciles à distinguer de la végétation naturelle. Toutefois, ces superficies cultivées peuvent présenter des confusions avec les zones nues.

\section{Données de végétation \\ Données structurales et fonctionnelles des glacis du bassin versant du Gourouol}

En vue de caractériser la structure et le fonctionnement des différents glacis, des inventaires floristiques, dendrométriques et de régénération ont été effectués courant mi-août à septembre 2012 sur des glacis très dégradés, des glacis moyennement dégradés et des glacis peu dégradés. Les données ont été récoltées suivant un échantillonnage stratifié et aléatoire. Le critère de stratification est le type de glacis. La taille des placeaux a été de $50 \mathrm{~m}$ x $20 \mathrm{~m}$ pour les ligneux, $10 \mathrm{~m}$ x $10 \mathrm{~m}$ pour les herbacées et $5 \mathrm{~m}$ x $5 \mathrm{~m}$ pour les individus juvéniles. L'inventaire floristique a été fait suivant l'approche phytosociologique de Braun-Blanquet (1932). Dans chaque placeau ligneux, les espèces présentes ont été recensées et affectées d'un coefficient d'abondance-dominance. Tous les individus ligneux dont le diamètre à $1,30 \mathrm{~m}$ du sol, est supérieur à $5 \mathrm{~cm}$ y ont été mesurés. A l'intérieur du placeau ligneux ont été installées des placettes herbacées et de régénération respectivement pour l'inventaire des herbacées et le comptage des individus juvéniles. Au total, 18 relevés ligneux, 18 
relevés herbacés, 18 relevés dendrométriques et 18 relevés de régénération ont été effectués par stade de dégradation du glacis. Ont été notés au cours de chaque relevé dendrométrique, le diamètre, la hauteur, le diamètre du houppier des ligneux ainsi que le nombre d'individus morts.

\section{Données environnementales des glacis du bassin versant du Gourouol}

Pendant les relevés, les variables environnementales de dégradation que sont le pourcentage de sol nu, les traces d'animaux, la sévérité de l'érosion (hydrique et éolienne), la quantité de matière organique du sol (litière) et le pourcentage d'individus ligneux rabougris ont été visuellement estimés puis codifiés selon l'échelle suivante: 1: variable absente; 2: variable peu abondante et 3: variable abondante. L'ensablement et la présence de rigoles ont servi pour estimer respectivement la sévérité de l'érosion éolienne et hydrique (Fatunbi et al., 2008).

\section{Analyse des données}

\section{Analyse des données satellitaires}

L'analyse de l'image satellitaire à partir des logiciels Erdas Imagine version 9.2 et ArcGIS.10 a permis la cartographie synchronique de l'Indice Normalisé Différentiel de la Végétation (NDVI) et de quantifier la superficie des stades de dégradation $\mathrm{du}$ bassin versant. Cette quantification tient compte du nombre de pixels. Le NDVI, basé sur l'activité photosynthétique est communément extrait des données satellitaires disponibles telles que les images MODIS et les images LandSat. Il est le plus utilisé dans le cadre des études sahéliennes pour la quantification de la couverture végétale pour le suivi de la végétation (Bénié et al., 2005).

Il s'exprime par la formule suivante:

$$
\mathrm{NDVI}=\frac{\mathrm{PIR}-\mathrm{BR}}{\mathrm{PIR}+\mathrm{BR}}
$$

PIR correspond à la réflectance des bandes dans le proche infra-rouge et $\mathrm{BR}$ correspond à la réflectance des bandes dans le rouge.
Le NDVI permet de montrer la variation spatiale d'un écosystème à un instant t donné (variation synchronique) selon la productivité de la végétation. Les résultats issus de l'analyse satellitaire ont été validés sur le terrain à travers la sélection randomisée de dix et huit coordonnées par stade de dégradation des glacis du bassin versant du Gourouol.

\section{Analyse des données structurales et fonctionnelles des glacis du bassin versant du Gourouol}

Les relevés phytosociologiques ont été soumis à une ordination via une DCA (Detrended Correspondence Analysis) à l'aide du logiciel Pc.ord.6 afin de cerner la distribution des placeaux en communautés selon le stade de dégradation. La caractérisation floristique des glacis s'est appuyée sur l'évaluation des attributs vitaux des écosystèmes (Aronson et al., 1995). Parmi les nombreux attributs vitaux proposés par ordre d'importance par Aronson et al. (1995), les plus pertinents sont: la richesse floristique en espèces pérennes, la richesse floristique en espèces annuelles, le spectre biologique, l'indice d'équitabilité de Piélou, les diversités $\alpha$ (indice de Shannon) et $\beta$ (indice de Sørensen) des végétaux et le taux de recouvrement total. Les équations suivantes ont servi pour le calcul des différents indices.

Indice de diversité de Shannon

$$
\mathrm{H}^{r}=-\sum_{\mathrm{i}=1}^{\mathrm{S}} \mathrm{pi} \ln (\mathrm{pl})
$$

Indice d'équitabilité de Piélou

$$
\mathbf{E}=\mathrm{H}^{\prime} / \ln (\mathrm{s})
$$

Indice de Sørensen

$$
\mathrm{cs}=\frac{2 J}{2 \prod+\mathrm{a}+\mathrm{b}}
$$

$P i$ est le quotient du recouvrement de l'espèce $\mathrm{i}$ dans le groupement par la somme de tous les recouvrements de toutes les espèces constitutives du groupement, ln est logarithme népérien;

$\mathrm{S}$ représente le nombre total d'espèces constitutives du groupement. L'indice de Shannon (H') varie de 0 à $\ln (\mathrm{S})$. H' tend vers 
0 lorsque le groupement est peu diversifié (renferme très peu d'espèces); H' est maximum quand le nombre d'espèces est élevé. Le mode de répartition des espèces au sein des groupements a été calculé à l'aide de l'indice d'équitabilité de Piélou (E). L'indice de Piélou varie de 0 à 1 . E tend vers 0 quand la quasi-totalité des effectifs est concentrée sur une espèce, et vers 1 lorsque toutes les espèces ont la même abondance. L'indice de Sørensen (Cs) mesure la similarité entre deux communautés. Il varie de 0 à 1 . Il tend vers 1 quand les communautés sont similaires; dans le cas contraire, elles ne le sont pas.

Dans la formule de l'indice de Sørensen, j est le nombre d'espèces communes aux communautés, a le nombre d'espèces rencontrées uniquement dans la communauté $\mathrm{A}$ et $\mathrm{b}$ le nombre d'espèces rencontrées uniquement dans la communauté B.

Pour chaque stade de dégradation, la densité des ligneux, le nombre de tiges, le nombre moyen d'individus juvéniles, le diamètre moyen des ligneux, la surface terrière, le nombre d'individus ligneux morts, la hauteur moyenne des individus ont été évalués.

\section{Analyse des données environnementales du bassin versant du Gourouol}

La quantification des variables par stade de dégradation a été faite suivant une transformation basée sur la contribution théorique relative de la variable. Ainsi, pour chacune des variables, la plus forte valeur observée a été retenue et divisée par les autres valeurs observées de la variable. La sommation des observations (si) a été ainsi effectuée par variable. Une moyenne exprimée en pourcentage ( $\mathrm{Sv}$ ) est calculée sur l'ensemble des observations (n) de la variable. A cet effet, la formule suivante a été utilisée:

$\mathrm{Sv}=\sum_{1-1}^{\mathrm{n}} \frac{\mathrm{si}}{\mathrm{n}}$

\section{Analyse statistique des données structurales, fonctionnelles et environnementales}

Le logiciel R-2.15.3 a été utilisé pour la comparaison des variables à travers le test non-paramétrique de Kruskal-Wallis entre les stades de dégradation et pour des analyses corrélatives. Une analyse en composantes principales (ACP) a été faite pour mettre en évidence les plus importantes et significatives variables intervenant dans la différenciation des stades de dégradation des glacis de la zone d'étude. L'ACP est une méthode mathématique utilisée pour réduire $p$ variables corrélées en un nombre q de variables non corrélées en exploitant le calcul matriciel et l'analyse des vecteurs et des valeurs propres (Biro et al., 2011). La mesure de la qualité de représentation des données est effectuée à l'aide du calcul de la contribution de l'inertie de chaque composante à l'inertie totale. L'analyse expose la contribution des individus et celle des variables aux composantes principales dans des espaces différents.

\section{RESULTATS}

Typologie et quantification de la dégradation de la végétation du bassin versant du Gourouol

Trois stades de dégradation de la végétation ont été diagnostiqués à partir d'imagerie satellitaire et d'observations de terrain dans le bassin versant. Il s'agit du stade très dégradé caractérisé par un faible NDVI et une couverture végétale inférieure à $10 \%$, le stade moyennement dégradé avec un NDVI moyen et une couverture végétale comprise entre $10 \%$ et $30 \%$ et le stade peu dégradé avec un NDVI élevé et une couverture végétale supérieure à 30\% (Tableau 1 et Figure 1).

Dans le bassin versant du Gourouol, les formations végétales moyennement dégradées sont les plus représentées $(45,95 \%)$. Elles sont suivies par les formations très dégradées $(27,41 \%)$ et les formations peu dégradées $(26,62 \%)$ (Figure 2).

La carte typologique et les observations de terrain montrent que les formations 
végétales du bassin versant du Gourouol en général et celles des glacis en particulier se subdivisent en formations de glacis très dégradées, en formations de glacis moyennement dégradées et en formations de glacis peu dégradées dont la caractérisation fonctionnelle et structurale s'avère importante.

\section{Caractérisation floristique des glacis du bassin versant du Gourouol suivant le stade de dégradation}

Le résumé de la DCA appliquée à la matrice de 54 relevés $x 56$ espèces montre que les deux premiers axes expliquent la variance à $56,3 \%$ et les trois premiers axes l'expliquent à 56,3\%. L'axe 3 n'explique donc pas la variance. On dénombre au total 56 espèces réparties en trois blocs (Figure 3); le bloc de relevés des glacis très dégradés avec une richesse floristique de 20 espèces réparties en 15 familles et 19 genres, le bloc de relevés des glacis moyennement dégradés avec une richesse spécifique de 26 espèces réparties en 16 familles et 23 genres et le bloc de relevés des glacis peu dégradés avec une richesse spécifique de 46 espèces reparties en 19 familles et 41 genres. L'analyse de l'indice de Sørensen montre une dissimilarité significative entre les différents stades de dégradation des glacis (Cs compris entre $82,79 \%$ et $98,36 \%$ ). Les paramètres de diversité ainsi que le recouvrement moyen décroissent des glacis peu dégradés vers les glacis très dégradés (Tableau 2). Les glacis peu dégradés présentent la plus forte diversité $\left(H^{\prime}=2,413 \pm 0,015\right)$ et la meilleure équitabilité $(\mathrm{E}=0,626 \pm 0,004)$ suivi par les glacis moyennement dégradés avec une diversité de Shannon de $1,584 \pm 0,018$ et un indice de diversité de Piélou de 0,486 $\pm 0,005$ et enfin le stade très dégradé dont l'indice de diversité de Shannon est de $1,188 \pm 0,018$ et l'indice d'équitabilité de Piélou de 0,265 $\pm 0,004$.
Espèces indicatrices des glacis du bassin versant du Gourouol suivant le stade de dégradation

L'analyse phytosociologique a permis d'identifier les espèces indicatrices suivant chaque stade de dégradation du glacis. Le Tableau 3 montre que les glacis peu dégradés se caractérisent par une abondance d'espèces indicatrices et que les glacis très dégradés et les glacis moyennement dégradés se caractérisent par un faible nombre d'espèces indicatrices.

Caractérisation du mode de vie des espèces en fonction du stade de dégradation des glacis du bassin versant du Gourouol

L'analyse des types biologiques montre la dominance des thérophytes dans tous les stades de dégradation des glacis (Figure 4). Ils représentent $80,76 \%$ pour le spectre brut et $98,21 \%$ pour le spectre pondéré dans les glacis très dégradés; $75,86 \%$ pour le spectre brut et $80,76 \%$ pour le spectre pondéré dans les glacis moyennement dégradés et $64,71 \%$ pour le spectre brut et $61.27 \%$ pour le spectre pondéré dans les glacis peu dégradés. Les thérophytes sont suivis des phanérophytes qui occupent $3,89 \%$ pour le spectre brut et $0,19 \%$ pour le spectre pondéré dans les glacis très dégradés; $20,68 \%$ pour le spectre brut et $19,16 \%$ pour le spectre pondéré dans les glacis moyennement dégradés et $26,31 \%$ pour le spectre brut et $37,85 \%$ pour le spectre pondéré dans les glacis peu dégradés. On note que les glacis très dégradés sont presque dépourvus de phanérophytes et que la quasitotalité des espèces qui $\mathrm{y}$ sont présentes est constituée par des thérophytes. Les autres types biologiques que sont les chamaephytes, les hémicryptophytes, les géophytes et les hélophytes sont très faiblement représentés dans tous les stades de dégradation des glacis. 
Caractérisation des variables de dégradation des glacis selon les stades de dégradation

L'analyse des variables de dégradation montre différentes tendances (Tableau 4). Les unes régressent des glacis peu dégradés aux glacis très dégradés. Il s'agit du recouvrement moyen des espèces, de la hauteur moyenne des espèces, du nombre de juvéniles par hectares, de la densité des ligneux, du diamètre moyen du houppier des espèces, de la quantité de litière, des individus ligneux rabougris et du nombre de tige des espèces. D'autres augmentent des glacis peu dégradés aux glacis très dégradés. Il s'agit de l'altitude, du pourcentage de sol nu et du taux d'érosion. Enfin, d'autres présentent un taux légèrement plus élevé dans les glacis moyennement dégradés; C'est le cas des individus morts, des traces d'animaux, de la surface terrière et du diamètre moyen des espèces. Le test de
Kruskal-Wallis appliqué aux variables exprime une différence significative pour la majorité des variables.

Analyse en composantes principales et répartition des variables selon le stade de dégradation des glacis du bassin versant du Gourouol

Le Tableau 5 présente les valeurs propres et les pourcentages des variables correspondant aux composantes principales. Les trois premières composantes représentent $69,8 \%$ de la variance totale. Les corrélations entre les trois premières composantes et les variables mesurées dans les stades de dégradation des glacis sont décrites par la Figure 5 (a-b). De même, la contribution des stades de dégradation aux trois premières composantes est présentée par la Figure 6 (ab).

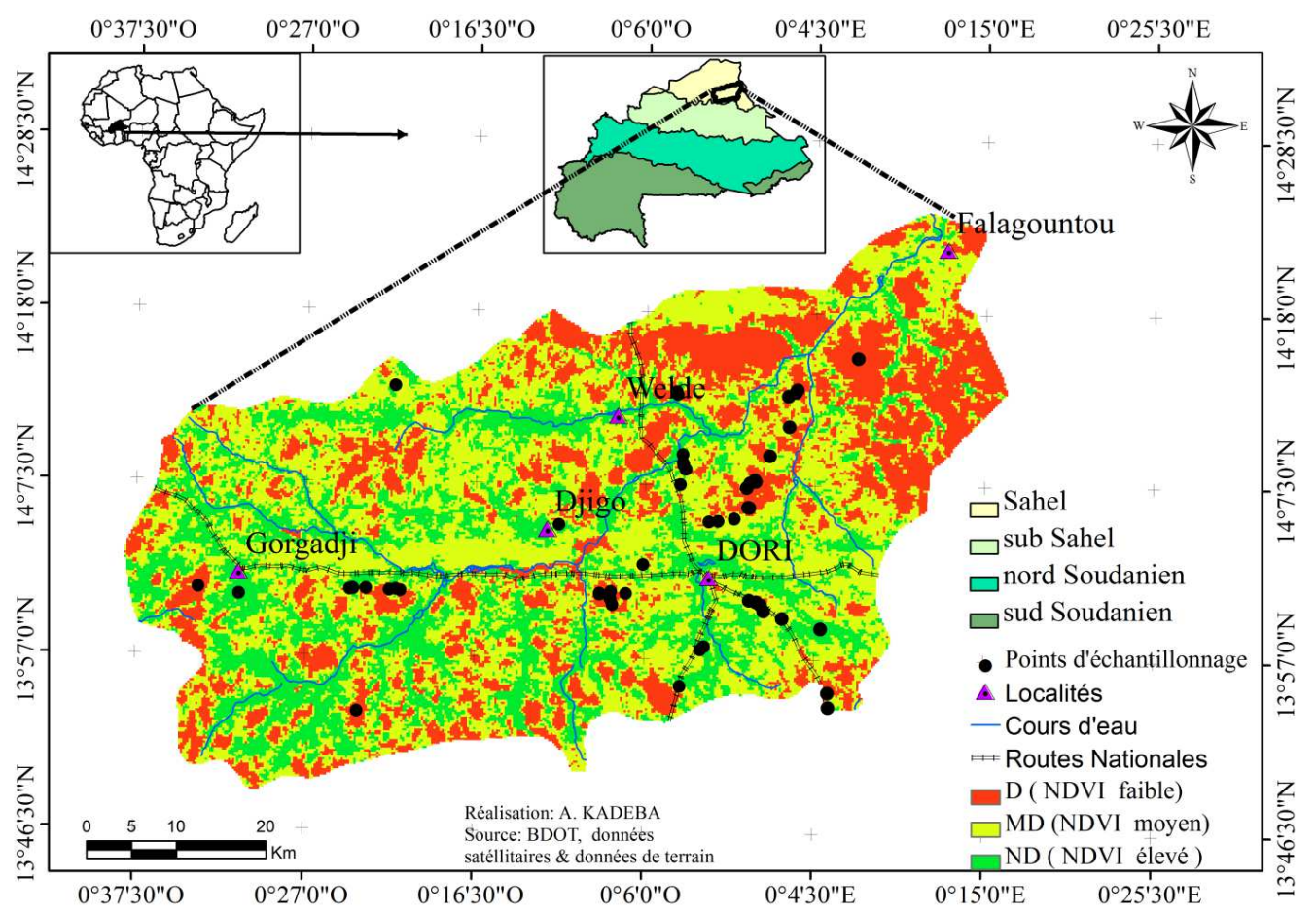

Figure 1: Localisation du bassin versant du Gourouol et typologie de ses stades de dégradation. $\mathrm{D}$, stade très dégradé; MD, stade moyennement dégradé; ND, stade peu dégradé; NDVI, Indice Normalisé de la Végétation. 


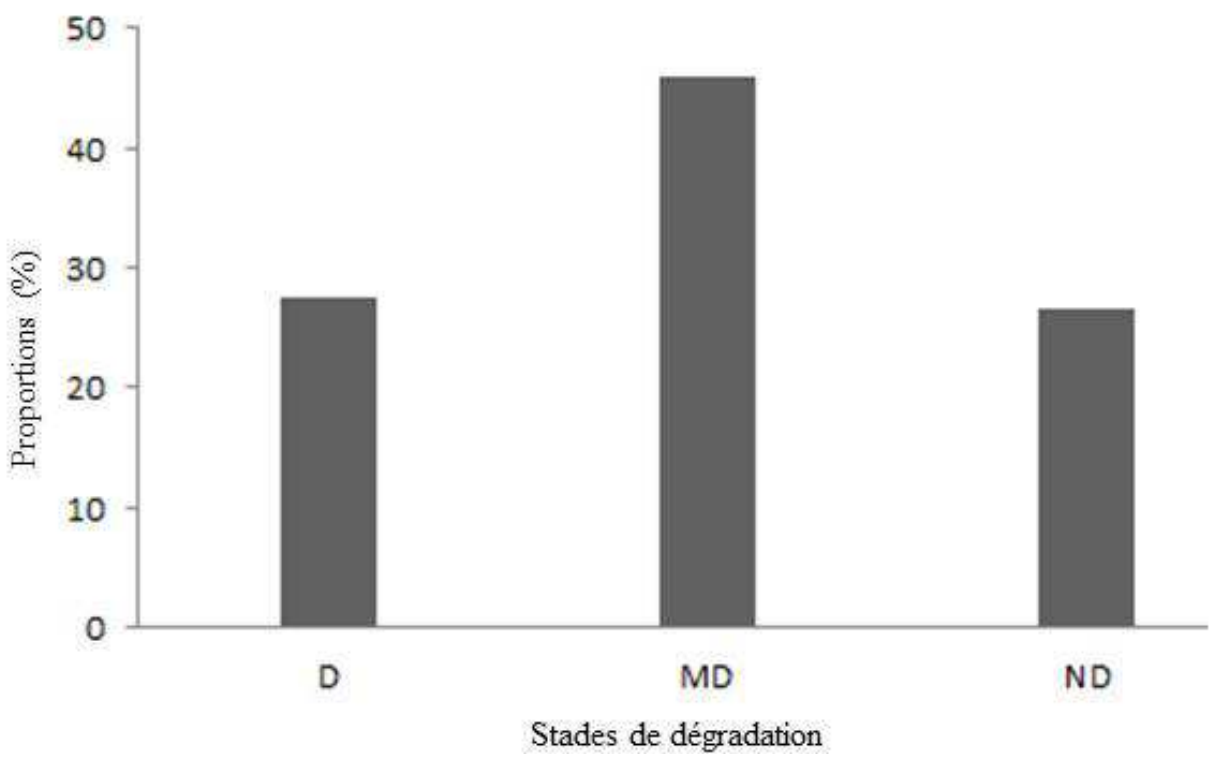

Figure 2: Proportions des différents stades de dégradation dans le bassin versant du Gourouol. $\mathrm{D}$, stade très dégradé; $\mathrm{MD}$, stade moyennement dégradé; $\mathrm{ND}$, stade peu dégradé.

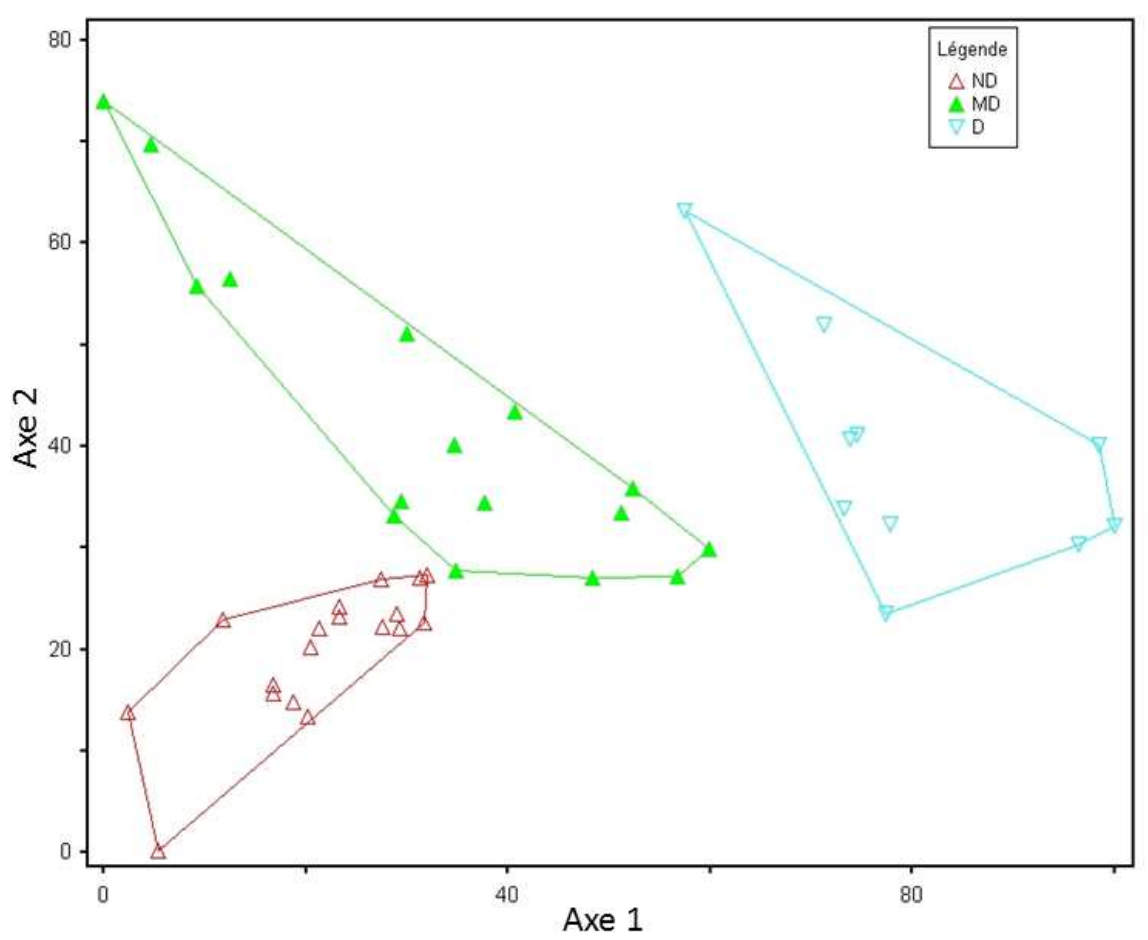

Figure 3: Diagramme d'ordination des relevés en communautés en fonction du stade de dégradation du glacis. L'ordination est basée sur 54 relevées (18 relevés par stade de dégradation du glacis) et 56 espèces dont 47 espèces sur les glacis peu dégradés (ND), 26 sur les glacis moyennement dégradés (MD) et 20 sur les glacis très dégradés $(\mathrm{D})$. Longueur du premier axe: 04,23, variance expliquée: $37 \%$. Longueur du second axe: 03,41, variance expliquée: $19,3 \%$. 
Glacis très dégradé

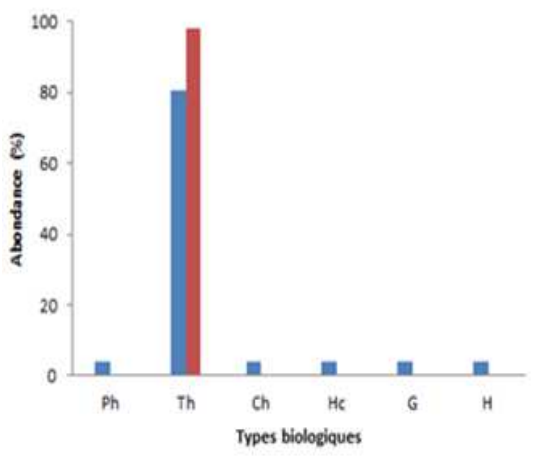

Glacis moyennement dégradé

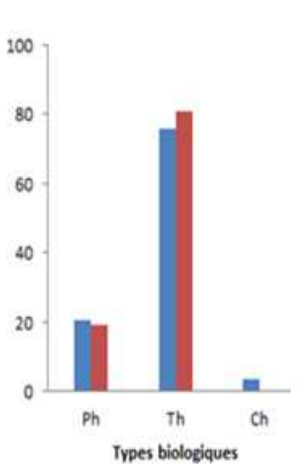

Glacis peu dégradé

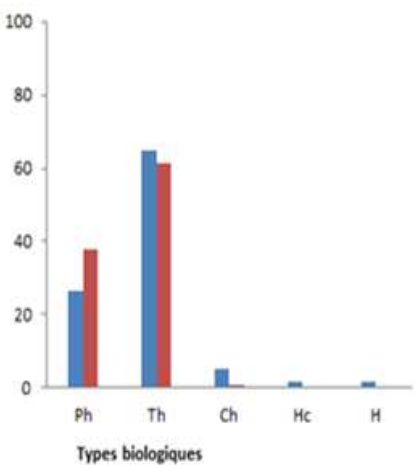

- Specte brut

a spectre pondété

Figure 4: Spectres biologiques des espèces dans les stades de dégradation des glacis. Ch: chamaephytes, G: géophytes, Hc: hémicryptophytes, Th: thérophytes, Ph: phanérophytes, H: hélophytes.
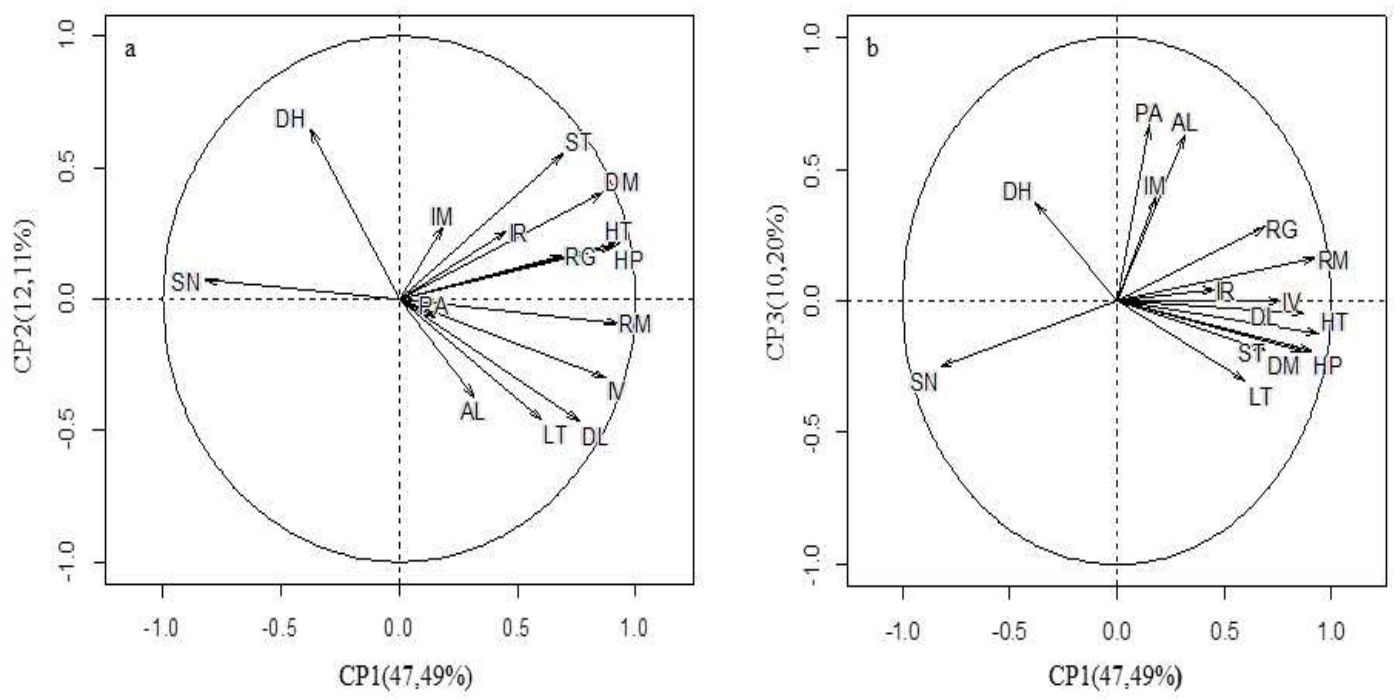

Figure 5: Contribution des variables de dégradation aux trois premières composantes principales. (a) $\mathrm{CP} 1 \times \mathrm{CP} 2$ et (b) $\mathrm{CP} 1 \times \mathrm{CP} 3$. DH, érosion hydrique et éolienne; SN, sol nu; IM, individus morts; PA, piétinements des animaux; IR, individus rabougris; RG, nombre moyen de juvéniles; AL, altitude moyenne; ST, surface terrière moyenne; LT, litière; HP, houppier moyen; HT, hauteur moyenne; IV, indice normalisé de végétation; RM, recouvrement moyen; DM, diamètre moyen; DL, densité moyenne des ligneux. 

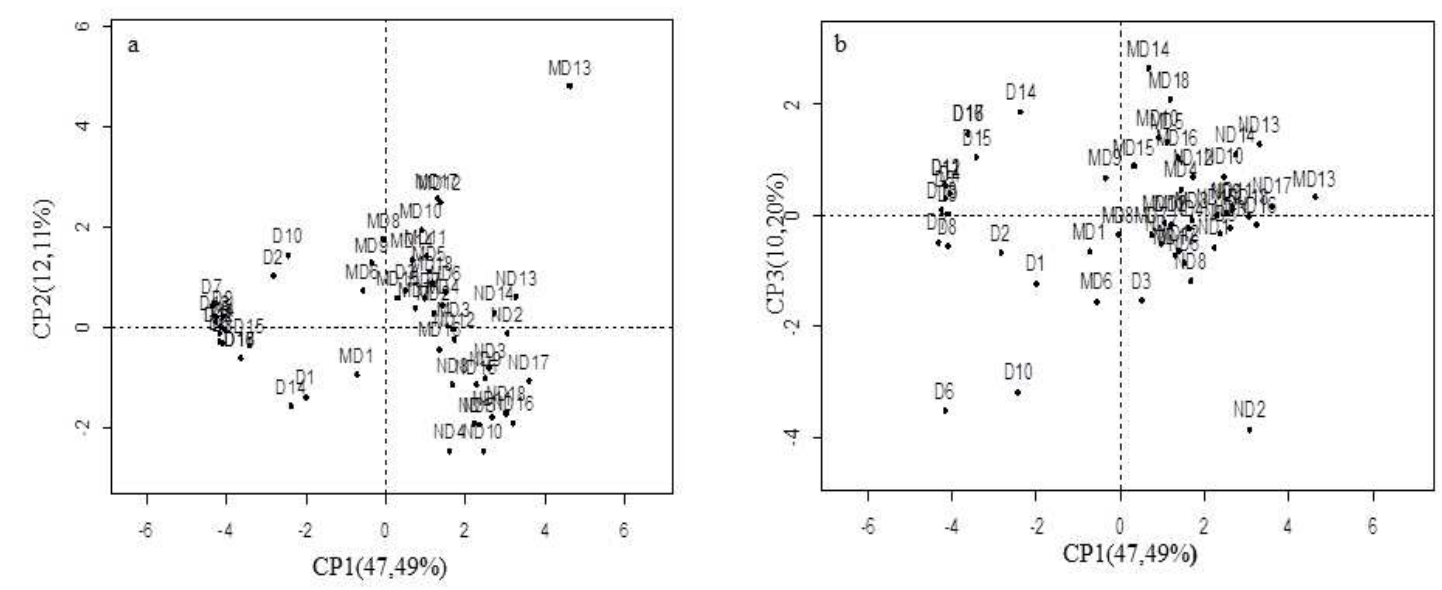

Figure 6: Contribution des stades de dégradation aux trois premières composantes principales. (a) $\mathrm{CP} 1 \times \mathrm{CP} 2$ et (b) $\mathrm{CP} 1 \times \mathrm{CP}$, (D1-D18), groupe de relevés des glacis très dégradés, (MD1-MD18) groupe de relevés des glacis moyennement dégradés et (ND1-ND18), groupe de relevés des glacis peu dégradés.

Tableau 1: Typologie des stades de dégradation selon l'imagerie satellitaire et la couverture végétale.

\begin{tabular}{lcc}
\hline $\begin{array}{l}\text { Stades de degradation de la végétation du } \\
\text { bassin versant de Gourouol }\end{array}$ & NDVI & Couverture végétale observée \\
\hline Très dégradé & Faible & Couverture végétale $<10 \%$ \\
Moyennement dégradé & Moyen & $10 \% \leq$ Couverture végétale $\leq$ \\
& Elevé & Couverture végétale $>30 \%$ \\
\hline Peu dégradé & &
\end{tabular}

Tableau 2: Résumé des paramètres de diversité suivant le stade de dégradation des glacis du bassin versant du Gourouol.

\begin{tabular}{|c|c|c|c|c|c|c|c|}
\hline $\begin{array}{l}\text { Stades de dégradation } \\
\text { des glacis du bassin } \\
\text { versant de Gourouol }\end{array}$ & $\mathbf{S}$ & $\mathbf{G}$ & $\mathbf{F}$ & $\operatorname{Ln}(S)$ & $\mathbf{E} \pm \mathbf{S E}$ & $\mathrm{H}^{\prime} \pm \mathrm{SE}$ & $\mathrm{SR} \pm \mathrm{SE}$ \\
\hline Peu dégradé & 47 & 41 & 19 & 3,8501 & $0,626 \pm 0,004$ & $2,413 \pm 0,015$ & $122,83 \pm 29,86$ \\
\hline Moyennement dégradé & 26 & 23 & 16 & 3,258 & $0,486 \pm 0,005$ & $01,584 \pm 0,018$ & $34,07 \pm 16,42$ \\
\hline Très dégradé & 20 & 19 & 15 & 2,995 & $0,265 \pm 0,004$ & $01,188 \pm 0,018$ & $14,85 \pm 08,28$ \\
\hline P-value & & & & & $0,03461 *$ & $0,03567 *$ & $0,01419^{*}$ \\
\hline
\end{tabular}

S, richesse spécifique; G, genre; F, famille; $J$, indice de Piélou; $H^{\prime}$, indice de Shannon; SR, somme des recouvrements; SE, erreur standard; *, différences significatives au seuil de 5\% 
Tableau 3: Espèces indicatrices des stades de dégradation selon la valeur indicatrice.

\begin{tabular}{llcc}
\hline Stades de dégradation des glacis & Espèces indicatrices & IV(\%) & P-value \\
\hline & Acacia senegal (L.) Willd. & 93,3 & 0,0010 \\
& Chloris pilosa Schumach. & 50,9 & 0,0010 \\
& Digitaria horizontalis Willd. & 68,1 & 0,0010 \\
& Eragrostis pilosa (L.) P.Beauv. & 78,6 & 0,0010 \\
& Leptadenia hastata (Pers.) Decne. & 53,6 & 0,0010 \\
& Panicum laetum Kunth & 63,3 & 0,0010 \\
& Acacia tortilis (Forssk.) Hayne & 43,9 & 0,0020 \\
& Balanites aegyptiaca (L.) Delile & 47,0 & 0,0020 \\
& Cassia tora L. & 54,0 & 0,0020 \\
& Dactyloctenium aegyptium (L.) Willd. & 46,9 & 0,0040 \\
\hline \multirow{2}{*}{ Moyennementént dégradé } & Schoenefeldia gracilis Kunth & 19,4 & 0,0316 \\
& Tribulus terrestris L. & 16,5 & 0,0373 \\
\hline \multirow{2}{*}{ Très dégradé } & Caralluma adscendens (Roxb.) Haw. & 36,5 & 0,0170 \\
& Acalypha crenata Hochst.ex A.Rich & 36,5 & 0,0250 \\
\hline
\end{tabular}

Tableau 4: Quantification comparative des variables par stade de dégradation.

\begin{tabular}{|c|c|c|c|c|}
\hline $\begin{array}{l}\text { Variables de dégradation des } \\
\text { glacis du bassin versant de } \\
\text { Gourouol }\end{array}$ & Peu dégradé & $\begin{array}{l}\text { Moyennement } \\
\text { dégradé }\end{array}$ & Très dégradé & P-value \\
\hline NDVI & Elevé & Moyen & Faible & \\
\hline Nombre de relevés & 18 & 18 & 18 & \\
\hline Nombre de tiges & 634 & 212 & 15 & \\
\hline Nombre de juvéniles/ha & 104 & 117 & 02 & \\
\hline Altitude (m) & $280,5 \pm 02,05$ & $283,5 \pm 02,25$ & $286,94 \pm 03,48$ & $2,210^{-16^{*}}$ \\
\hline Sol nu (\%) & $35,27 \pm 07,73$ & $41,66 \pm 05,47$ & $90,66 \pm 02,53$ & $1,210^{-5^{*}}$ \\
\hline Individus morts (\%) & $09,41 \pm 05,51$ & $29,44 \pm 10,92$ & $00 \pm 00$ & $0,001926^{*}$ \\
\hline Individus rabougris (\%) & $16,66 \pm 06,51$ & $13,17 \pm 05,35$ & $00 \pm 00$ & $4,410^{-5^{*}}$ \\
\hline Erosion (\%) & $61,11 \pm 06,76$ & $87,96 \pm 0,05$ & $87,96 \pm 05,00$ & $0,002809 *$ \\
\hline Traces d'animaux (\%) & $96,29 \pm 03,70$ & $100 \pm 00$ & $88,88 \pm 07,62$ & 0,3402 \\
\hline Litière $(\%)$ & $77,77 \pm 06,02$ & $27,77 \pm 09,23$ & $11,11 \pm 07,62$ & $3,310^{-6^{*}}$ \\
\hline Surface terrière $\left(\mathrm{m}^{2} / \mathrm{ha}\right)$ & $03,55 \pm 0,02$ & $4,38 \pm 0,04$ & $0,032 \pm 0,02$ & $9,610^{-9 *}$ \\
\hline Diamètre moyen $(\mathrm{cm})$ & $15,48 \pm 0,82$ & $16,68 \pm 01,36$ & $2,81 \pm 01,41$ & $9,910^{-9 *}$ \\
\hline Houppier moyen (m) & $05,11 \pm 0,20$ & $04,77 \pm 0,24$ & $00.76 \pm 0,38$ & $5,310^{-8^{*}}$ \\
\hline Densité (Nombre de tige/ha) & $352,22 \pm 25,18$ & $117,77 \pm 08,87$ & $08.33 \pm 04,37$ & $6,410^{-11^{*}}$ \\
\hline Hauteur moyenne (m) & $03,85 \pm 0,11$ & $03,50 \pm 0,22$ & $0,47 \pm 0,23$ & $1,8510^{-8 *}$ \\
\hline Recouvrement moyen (\%) & $92,38 \pm 0,016$ & $77,77 \pm 0,034$ & $23,33 \pm 0,06$ & $6,910^{-8^{*}}$ \\
\hline
\end{tabular}


Tableau 5: Valeurs propres extraites de la matrice de corrélation des variables mesurées.

\begin{tabular}{lccc}
\hline $\begin{array}{l}\text { Composantes principales } \\
\text { (CP) }\end{array}$ & $\begin{array}{c}\text { Valeurs } \\
\text { propres }\end{array}$ & $\begin{array}{c}\text { Pourcentages de la } \\
\text { variance par CP }\end{array}$ & $\begin{array}{c}\text { Pourcentages cumulés } \\
\text { de la variance totale }\end{array}$ \\
\hline 1 & 7,12 & 47,49 & 47,49 \\
2 & 1,81 & 12,11 & 59,6 \\
3 & 1,52 & 10,20 & 69,8 \\
4 & 1,03 & 6,88 & 76,69 \\
5 & 0,89 & 5,96 & 82,65 \\
6 & 0,82 & 5,52 & 88,17 \\
7 & 0,49 & 3,32 & 91,49 \\
8 & 0,38 & 2,54 & 94,04 \\
9 & 0,33 & 2,25 & 96,3 \\
10 & 0,23 & 1,54 & 97,84 \\
11 & 0,12 & 0,84 & 98,69 \\
12 & 0,09 & 0,63 & 99,32 \\
13 & 0,06 & 0,4 & 99,72 \\
14 & 0,02 & 0,19 & 99,92 \\
15 & 0,01 & 0,07 & 100 \\
\hline
\end{tabular}

\section{DISCUSSION}

Typologie de la dégradation de la végétation du bassin versant du Gourouol

La végétation du secteur sahélien du Burkina Faso montre une diversité de faciès exprimée à travers l'imagerie satéllitaire appuyée d'observations visuelles et de mesures de terrain. Cette diversité de faciès s'exprime aussi bien entre les unités géomorphologiques qu'au sein d'une même unité géomorphologique. Cela confirme les travaux de Jacqueminet (1991) qui, dans ces études au Sahel du Burkina Faso a prouvé l'existence d'unités paysagiques au sein d'une même unité géomorphologique et entre plusieurs unités géomorphologiques. La catégorisation du NDVI combinées aux observations visuelles de la végétation montre un gradient de dégradation basé sur trois stades de dégradation; le stade très dégradé, le stade moyennement dégradé et le stade peu dégradé. Des études antérieures ont utilisé la variation du taux de couverture végétale pour asseoir et caractériser des stades de dégradation (Al-Awadhi et al., 2005; Ingram et al., 2005; Wessels et al., 2004). La forte représentativité des stades très dégradé et moyennement dégradé s'explique par le fait que ces stades prennent en compte les superficies cultivées, unités d'occupation des terres difficilement séparables des zones nues. Les trois stades de dégradation de la végétation sahélienne observés sont aussi perceptibles sur les glacis du bassin versant de Gourouol.

Caractérisation structurale et fonctionnelle des stades de dégradation des glacis du bassin versant du Gourouol
L'ordination
des
relevés phytosociologiques des glacis montre que des communautés bien distinctes se développent en fonction du stade de dégradation du glacis. La diversité floristique ainsi que le taux de couverture végétale y augmentent des glacis très dégradés aux glacis peu dégradés. Cette variation de la diversité floristique selon le stade de dégradation s'explique par les 
activités humaines dont le surpâturage et l'agriculture, principales causes de la catégorisation des stades de dégradation (Bénié et al., 2005). Les indices d'équitabilité de Piélou montrent que la quasi-totalité des espèces sur les glacis dégradés et les glacis très dégradés est concentrée sur une seule espèce tandis que sur les glacis peu dégradés, les espèces ont presque la même abondance. Il découle de l'analyse floristique qu'il existe des espèces indicatrices des trois stades de dégradation des glacis. La caractérisation des formes de vie montre la dominance des thérophytes (espèces annuelles) dans tous les stades de dégradation dont l'abondance augmente du stade très dégradé au stade peu dégradé. Ce résultat confirme les travaux de Schmidt et al. (2008) qui dans leurs études au sahel du Burkina Faso, ont montré la dominance des thérophytes. Par ailleurs, on note la présence des phanérophytes dont l'abondance décroît du stade peu dégradé au stade très dégradé. Ces phanérophytes sont presque inexistants dans le stade très dégradé. Ces résultats corroborent ceux de Jauffret (2001) qui a mentionné en Tunisie que les thérophytes sont des indicateurs des stades de dégradation. Cet auteur a par ailleurs noté que la thérophytie est une stratégie d'adaptation en région aride car dépassant la sécheresse sous forme de graines. La même observation a été notée par Aronson et al. (1995) qui ajoutent que l'abondance des espèces pérennes dans un écosystème indique sa stabilité tandis que l'abondance des espèces annuelles indique son aridité. Nos résultats expriment que la dégradation des écosystèmes se manifeste par une régression des espèces pérennes en général, les phanérophytes, suivi de leur remplacement par des espèces annuelles que sont les thérophytes. Ce qui soutient les travaux de Schmidt et al. (2005). Ces auteurs ont noté que la forte dominance des espèces annuelles dans le Sahel du Burkina Faso traduit son aridité.
Analyse à composantes principales et répartition des variables en fonction du stade de dégradation des glacis du bassin versant du Gourouol

L'analyse des variables de dégradation selon les stades de dégradation des glacis du bassin versant de Gourouol montre en général une différence significative entre celles des glacis très dégradés et celles des glacis peu dégradés sauf dans le cas des traces d'animaux où on n'observe pas de différences significatives. Cela se justifie par le fait que tous les glacis sahéliens sont pâturés. La valeur élevée de la surface terrière dans les glacis moyennement dégradés s'explique par les compétitions interspécifique et intraspécifique qui sont moindres dans ce stade de dégradation entraînant la forte valeur du diamètre moyen et par conséquent la forte valeur de la surface terrière.

L'analyse en composantes principales montre que les trois stades de dégradation des glacis sont discriminés le long de CP1 principalement à cause de la forte corrélation positive du recouvrement moyen des espèces des glacis peu dégradés et de la forte corrélation négative du pourcentage de sol nu des glacis très dégradés. De plus, la Figure 5a montre que le recouvrement moyen des espèces et le pourcentage de sol nu sont dépendants de la première composante (CP1). On note également une faible corrélation positive du nombre d'individus morts avec la deuxième composante (CP2). Conséquemment, on observe que les glacis très dégradés et les glacis peu dégradés sont séparés par les glacis moyennement dégradés. En comparant les composantes CP1 et CP3, on s'aperçoit aussi clairement que les glacis très dégradés et les glacis peu dégradés sont séparés par les glacis moyennement dégradés. Cela s'explique principalement par la forte corrélation positive du degré de piétinements des animaux à CP3 d'une part et par la non corrélation de cette variable avec la composante CP1 d'autre part. 
Cette analyse statistique montre que le taux de couverture végétale augmente des glacis très dégradés aux glacis peu dégradés, contrairement au pourcentage de sol nu qui décroît des glacis très dégradés aux glacis peu dégradés. Ainsi, les glacis très dégradés se caractérisent par un pourcentage de sol nu élevé et un fort degré d'érosion (éolienne et hydrique). Les glacis peu dégradés se caractérisent par des arbres à gros houppiers, un taux de recouvrement élevé, une forte surface terrière, un pourcentage de litière élevé, des espèces à hauteur assez élevée, une abondance d'individus juvéniles et un indice de végétation élevé. Les glacis moyennement dégradés se caractérisent par la présence d'individus morts et une forte présence de traces d'animaux. Ces résultats sont en accord avec ceux de Fatunbi et al. (2008) qui ont souligné le rôle capital de la couverture végétale pour la prévention de la dégradation des terres.

\section{Conclusion}

A travers l'imagerie satellitaire et les observations de terrain, trois stades de dégradation ont été mis en évidence au sahel en général et sur les glacis sahéliens du Burkina Faso en particulier. Ce sont le stade très dégradé dont la couverture végétale est faible (inférieures à 10\%), le stade moyennement dégradé dont la couverture végétale est comprise entre $10 \%$ et $30 \%$ et le stade peu dégradé avec une forte couverture végétale (supérieures à 30\%). Il existe des différences significatives entre les stades de dégradation tant sur le plan fonctionnel que structural. L'indice de Sørensen (diversité- $\beta$ ) montre une dissimilarité entre les stades de dégradation. Cette dissimilarité a été approuvée par les espèces indicatrices, l'indice de Shannon (diversité- $\alpha$ ) et les formes de vie dans chaque stade de dégradation. Dans ce sens on note que la dégradation se manifeste par une thérophytie qui s'accentue du stade peu dégradé au stade très dégradé.

L'analyse en composantes principales permet de retenir des variables indicatrices de chaque stade de dégradation. Il s'agit du houppier, du taux de recouvrement, de la surface terrière, de la litière, de la hauteur des espèces, des juvéniles et de l'indice normalisé de végétation qui présentent de fortes valeurs dans les glacis peu dégradés; du pourcentage de sol nu et du degré d'érosion qui ont de forts taux dans les glacis très dégradés; des individus morts et des traces d'animaux qui dominent dans les glacis moyennement dégradés.

Cette étude constitue un outil d'aide de décision et d'orientation des différents programmes de restauration et d'aménagement de la végétation sahélienne.

\section{REMERCIEMENTS}

Cette étude a été conduite grâce au projet UNDESERT (Understanding and Combating desertification to mitigate its impact on Ecosystem services), financé par l'Union Européenne (EU FP7 243906). Nous exprimons notre gratitude aux populations de la ville de Dori en général et particulièrement aux guides de terrain Sambo CISSE et Ibrahim DIALLO pour leurs aides à la récolte des données de terrain.

\section{REFERENCES}

Agnew CT, Chappel A. 2000. Drought in the Sahel. GeoJournal, 48: 299-311.

Al-Awadhi JM, Omar SA, Misak RF. 2004. Land degradation indicators in Kuwait. Land Degradation \& Development, 16: 163-176.

Aronson J, Floret C, Le Floc'h E, Ovalle C, Pontanier R. 1995. Restauration et Réhabilitation des Ecosystèmes Dégradés en Zones Arides et Semi-Arides. Le Vocabulaire et les Concepts. John Libbey Eurotext : Paris ; 11-29.

Barral H. 1967. Les populations d'éleveurs et les problèmes pastoraux dans le nord-est de la Haute-Volta (cercle de DoriSubdivision de l'Oudalan). Cah. Orstom. Sér. Sci. Hum., 4(1): 30.

Benié GB, Kabore SS, Goïta K, Courel MF. 2005. Remote sensing-based spatio 
temporal modelling to predict biomass in sahelian grazing ecosystem. Ecological Modelling, 184: 341-354.

Benslimane M, Hamimed A, El Zerey W, Khaldi A, Mederbal K. 2008. Analyse et suivi du phénomène de la désertification en Algérie du Nord. VertigO, 8(3), http://vertigo.revues.org/6782.

Biro K, Pradhan B, Burchroithner M, Makeschin F. 2011. Land use/Land cover change analysis and its impact on soil properties in the northern part of Gadarif region, Sudan. Land Degradation \& Development, Doi: 10.1002/Idr. 1116.

Brabant P. 2010. Une méthode d'évaluation et de cartographie de la dégradation des terres. Proposition de directives normalisées. Les dossiers thématiques du CSFD, No8. CSFD / Agropolis International, Montpellier, France, $52 \mathrm{p}$.

Brabant P, Simonneaux V, Egue K. 1996. Etat actuel de dégradation des terres et de l'environnement au Togo déterminé à l'aide de la télédétection. Notice explicative de la carte des indices de dégradation. Coll. Note explicative. 112. Orstom, Paris, 57 p.

Braun-Blanquet J. 1932. Plant Sociology, the study of Plant Communities. McGray Hill : New York, London.

Cornet A. 2002. La désertification: un problème d'environnement, un problème de développement, résumé de la conférence à Agropolis Muséum. IRD, 8 p.

Fatunbi AO, Dube S. 2008. Land degradation evaluation in a game reserve in Eastern Cape of South Africa: soil properties and vegetation cover. Scientific Research and Essay, 3(3): 111-119.

Ganaba S. 2008. Caractérisation, utilisations, tests de restauration et gestion de la végétation ligneuse au sahel, Burkina Faso. Thèse de Doctorat d'Etat ès sciences Naturelles, $\mathrm{n}^{\circ} 117$, Université Cheikh Anta Diop de Dakar, Sénégal, 287 p.
Grouzis M. 1984: Restauration des pâturages: synthèse des travaux de reboisement dans la région de Markoye. Projet Fond Européen de développement (FED): développement de l'élevage dans l'organisme régional de développement (ORD) du sahel. Ouagadougou, centre Orstom, 39 p.

Grouzis M. 1983. Pâturages sahéliens du nord du Burkina Faso: capacité de charge, production fréquentielle et dynamique de la qualité fourragère. Projet Fond Européen de développement (FED): développement de l'élevage dans l'organisme régional de développement (ORD) du sahel. Ouagadougou, centre Orstom, 8 p.

Guinko S. 1984. Végétation de la haute-Volta. Thèse de Doctorat ès Sciences Naturelles, Université de Bordeaux III, France, vol 2, $394 \mathrm{p}$.

Hammad AA, Tumeizi A. 2010. Land Degradation: socioeconomic and environmental causes and consequences in the eastern Mediterranean. Land Degradation \& Development, Doi: 10: 1002/idr.1069.

Ingram CJ, Terance PD, Robert JW. 2005. Mapping tropical forest structure in south-eastern Madagascar using remote sensing and artificial neural networks. Remote Sensing of Environment, 94: 491507.

Jacqueminet C. 1991. Analyse de la structure spatiale du couvert ligneux en milieu sahélien sur images satellitaires spot (étude appliquée au secteur dunaire d'Oursi au Burkina Faso et au Ferlo sableux au Sénégal), Thèse de doctorat (nouveau régime), Ecole des hautes études en Sciences sociales, $224 \mathrm{p}$.

Jauffret S. 2001. Validation et comparaison de divers indicateurs des changements à long terme dans les écosystèmes méditerranéens arides: application au suivi de la désertification dans le sud tunisien. Thèse de doctorat. Université Aix-Marseille, 364 p. 
Kagambega WF, Traoré S, Thiombiano A, Boussim IJ. 2011. Impact de trois techniques de restauration des sols sur la survie et la croissance de trois espèces ligneuses sur les «zipéllés» au Burkina Faso. International Journal of Biological and Chemical Sciences, 5(3): 901-914.

Kosmidou V, Petrou Z, Bunce GHR, Mücher AC, Jongman HGR, Bogers MBM, Lucas MR, Tomaselli V, Blonda P, PadoaSchioppa E, Manakos I, Petrou M. 2014. Harmonization of the land covers classification system (LCCS) with the general habitat categories (GHC) classification system. Ecological Indicators, 36: 29-300.

Kiéma A, Nianogo AJ, Ouédraogo T. 2008. Effets des cordons pierreux sur la régénération d'un pâturage naturel de glacis au sahel. Cahiers Agricultures, 17(3): 281-288.

Maitima J, Robin SR, Louis NG, Amos M, Herbert L, Derek P, Simon M, Stephen M, Sam M. 2004. A methodological guide on how identify trends in the linkages between changes in land use, biodiversity and land degradation: the land use change, impacts and dynamics project, working paper number 43. International livestock research institute: $32 \mathrm{p}$.

Miguel GG, Fernando TM. 2011. Remote sensing data predict indicators of soil functioning in semi-arid steppes, Central Spain. Ecological Indicators, 11: 14761481.

Morsli B, Mazour M, Mededjel N, Hamoudi A, Roose E, 2004. Influence de l'utilisation des terres sur les risques de ruissellement et d'érosion sur les versants semi-arides du nord-ouest de l'Algérie. Sécheresse, 15(1): 96-104.
Peter IE, Alaci D. 2008. Analytical Situations of Land degradation and Sustainable management strategies in Africa. Journal of Agriculture and Social Sciences, 4: 4252.

Rakotoarimanana V, Gondard H, Ranaivoarivelo N, Carrière S. 2008. Influence du pâturage, sur la diversité floristique, la production et la qualité fourragères d'une savane, des Hautes Terres malgaches. Sécheresse, 19(1): 3946.

Roselt / Oss. 2004. Indicateurs écologiques. Une première approche méthodologique pour la surveillance de la biodiversité et des changements environnementaux. Collection ROSELT / OSS- Document Scientifique $\mathrm{n}^{\circ}$ 4: $78 \mathrm{p}$.

Schmidt M, König K, Müller JV. 2008. Modelling species richness and life form composition in Sahelian Burkina Faso with remote sensing data. Journal of Arid Environment, 72: 1506-1517.

Schmidt M, Kreft H, Thiombiano A, Zizka G. 2005. Herbarium collections and field data-based plant diversity maps for Burkina Faso. Diversity and Distributions, 11: 509-516.

Thiombiano A, Schmidt M, Dressler S, Ouédraogo A, Hahn K, Zizka G. 2012. Catalogue des plantes vasculaires du Burkina Faso. Boissiera, 65: 1-391.

Wessels KJ, Prince SD, Frost PE, Zyl VD. 2004. Assessing the effects of humaninduced land degradation in the former homelands of northern South Africa with a $1 \mathrm{Km}$ AVHRR NDVI time-series. Remote Sensing of Environments, 91: 4767. 